\title{
A Characterization of Ruled Real Hypersurfaces in Non-Flat Complex Space Forms
}

\author{
George Kaimakamis ${ }^{1,+}$, Konstantina Panagiotidou ${ }^{1,2, *,+}$ and Juan de Dios Pérez ${ }^{3,+}$ \\ 1 Faculty of Mathematics and Engineering Sciences, Hellenic Army Academy, Vari, \\ 16673 Attiki, Greece; gmiamis@sse.gr \\ 2 Department of Mathematics, School of Sciences, Aristotle University of Thessaloniki, \\ 54124 Thessaloniki, Greece \\ 3 Departmento de Geometria y Topologia, Universidad de Granada, 18071 Granada, Spain; jdperez@ugr.es \\ * Correspondence: konpanagiotidou@gmail.com \\ + These authors contributed equally to this work.
}

Received: 28 March 2020; Accepted: 17 April 2020; Published: 21 April 2020

\begin{abstract}
The Levi-Civita connection and the k-th generalized Tanaka-Webster connection are defined on a real hypersurface $M$ in a non-flat complex space form. For any nonnull constant $k$ and any vector field $X$ tangent to $M$ the $\mathrm{k}$-th $C$ ho operator $F_{X}^{(k)}$ is defined and is related to both connections. If $X$ belongs to the maximal holomorphic distribution $\mathbb{D}$ on $M$, the corresponding operator does not depend on $k$ and is denoted by $F_{X}$ and called Cho operator. In this paper, real hypersurfaces in non-flat space forms such that $F_{X} S=S F_{X}$, where $S$ denotes the Ricci tensor of $M$ and a further condition is satisfied, are classified.
\end{abstract}

Keywords: k-th generalized Tanaka-Webster connection; k-th Cho operator; real hypersurface; Ricci tensor; non-flat complex space form

\section{Introduction}

An n-dimensional Kähler manifold with constant holomorphic sectional curvature $c$ called complex space form. Depending on the value of the holomorphic sectional curvature, a complete and simply connected complex space form can be analytically isometric to a complex projective space $\mathbb{C} P^{n}$ if $c>0$, to a complex Euclidean space $\mathbb{C}^{n}$ if $c=0$, or to a complex hyperbolic space $\mathbb{C} H^{n}$ if $c<0$. In case of $\mathbb{C} P^{n} c$ is considered 4 and in case of $\mathbb{C} H^{n} c$ is equal to -4 . The term non-flat complex space forms and the symbol $M^{n}(c), n \geq 2$, is used to describe the complex projective and complex hyperbolic spaces, when it is not necessary to distinguish them.

We consider $M$ to be a connected real hypersurface of $M^{n}(c)$ without boundary. We denote $\nabla$ the Levi-Civita connection on $M$ and $J$ the complex structure of $M^{n}(c)$. Next we consider a locally defined unit normal vector field $N$ on the real hypersurface and we denote by $\xi=-J N$, which is the structure vector field on $M$ and it is tangent to $M$. The real hypersurface is a Hopf hypersurface, if the structure vector field is an eigenvector of the shape operator $A$ of the real hypersurface. Then the corresponding eigenvalue is denoted by $\alpha=g(A \xi, \xi)$. Furthermore, the Kähler structure of non-flat complex space induces on $M$ an almost contact metric structure $(\phi, \xi, \eta, g)$, where $\phi$ is the tangent component of $J$ and $\eta$ is an one-form given by $\eta(X)=g(X, \xi)$ for any $X$ tangent to $M$.

In [1-4], Takagi classified homogeneous real hypersurfaces in complex projective space into 6 types. Among them there are the following

- $\quad$ type $\left(A_{1}\right)$ real hypersurfaces that are geodesic hyperspheres of radius $r, 0<r<\frac{\pi}{2}$,

- type $\left(A_{2}\right)$ real hypersurfaces that are tubes of radius $r, 0<r<\frac{\pi}{2}$, over totally geodesic complex projective spaces $\mathbb{C} P^{k}, 0<k<n-1$ (type $\left(A_{1}\right),\left(A_{2}\right)$ are called type $(A)$ real hypersurfaces), 
- $\quad$ type $(B)$ real hypersurfaces that are tubes of radius $r, 0<r<\frac{\pi}{4}$, over the complex quadric.

The above are Hopf hypersurfaces whose principal curvatures are constant. In case of complex hyperbolic space, real hypersurfaces with constant principal curvatures were studied by Montiel in [5] and by Berndt in [6]. In this case the real hypersurfaces are divided into two types:

- $\quad$ type $(A)$ real hypersurfaces which are either a horosphere in $\mathbb{C} H^{n}$, or a geodesic hypersphere or a tube over a totally geodesic complex hyperbolic hyperplane $\mathbb{C} H^{n-1}$, or a tube over a totally geodesic $\mathbb{C} H^{k}(1 \leq k \leq n-2)$,

- type (B) real hypersurfaces which are tubes of radius $r>0$ over totally real hyperbolic space $\mathbb{R} H^{n}$.

The above real hypersurfaces are homogeneous and Hopf.

Ruled real hypersurfaces are another important class of real hypersurfaces in $M^{n}(c)$. They are constructed in the following way: we consider a regular curve $\gamma$ in a non-flat complex space form and $X$ a tangent vector field. Then at each point of the curve there is a unique hyperplane of $M^{n}(c)$ cutting the curve in a way to be orthogonal to both $X$ and JX. Then, the union of all these hyperplanes form the ruled hypersurface. The previous description is equivalent to the fact that on ruled hypersurfaces in $M^{n}(c)$ the maximal holomorphic distribution $\mathbb{D}$ of $M$ at any point, which includes all the vectors orthogonal to $\xi$, is integrable and it has as integrable manifold $M_{n-1}(c)$, i.e $g(A \mathbb{D}, \mathbb{D})=0$. An example of a ruled real hypersurface in $\mathbb{C} P^{n}$ is given by Kimura in [7]: Let $\mathbb{C}^{n+1}$ be an $n+1$-dimensional complex Euclidean space with the canonical coordinates $\left(z_{0}, z_{1}, \ldots, z_{n}\right)$ and $S^{2 n+1}$ a unit sphere in $\mathbb{C}^{n+1}$ with center at the origin. $S^{2 n+1}$ is principal fibre bundle over $\mathbb{C} P^{n}$ with structure group $S^{1}$ and projection map $\Pi$. Let $M^{\prime}$ the real hypersurface in $S^{2 n+1}$ given by

$$
\begin{gathered}
M^{\prime}=\left\{z=\left(r e^{i t} \cos (\theta), r e^{i t} \sin (\theta),\left(1-r^{2}\right)^{\frac{1}{2}} z_{2}, \ldots,\left(1-r^{2}\right)^{\frac{1}{2}} z_{n} \in \mathbb{C}^{n+1} ;\right.\right. \\
\left.\sum_{j=2}^{n}\left|z_{j}\right|^{2}=1,0<r<1,0 \leq t<2 \pi \text { and } 0 \leq \theta<2 \pi\right\} .
\end{gathered}
$$

Then $M=\Pi\left(M^{\prime}\right)$ is a minimal, ruled and no complete real hypersurface in $\mathbb{C} P^{n}$. (see also [8]).

The Jacobi operator $R_{X}$ with respect to a unit vector field $X$ is defined by $R_{X}=R(\cdot, X) X$, where $R$ is the curvature tensor field on $M$, which is a self-adjoint endomorphism of the tangent space. It is connected to Jacobi vector fields, which are solutions of the Jacobi equation $\nabla_{\dot{\gamma}}\left(\nabla_{\dot{\gamma}} Y\right)+R(Y, \dot{\gamma}) \dot{\gamma}=0$ along a geodesic $\gamma$ in $M$. The Jacobi operator with respect to the structure vector field $\xi$, $R_{\tilde{\zeta}}$, is called the structure Jacobi operator on $M$.

Let $R$ denote the Riemannian curvature tensor of $M$. Then the Ricci tensor is defined by

$$
S X=\sum_{i=1}^{2 n-1} R_{E_{i}}(X)=\sum_{i=1}^{2 n-1} R\left(X, E_{i}\right) E_{i}
$$

where $\left\{E_{i}\right\}_{i=1, \ldots, 2 n-1}$ is an orthonormal basis of $T M$, for any $X$ tangent to $M$. Many problems of classification of real hypersurfaces in non-flat complex space forms are related to their Ricci tensor.

In [9] is proved that real hypersurfaces in $M^{n}(c), n \geq 3$, do not admit parallel Ricci tensor (i.e., $\nabla_{X} S=0$, for any vector field $X$ tangent to $M$ ). In [10] the previous result is also proved for three dimensional real hypersurfaces.

As a consequence real hypersurfaces satisfying weaker conditions than the parallelism of $S$ are studied. First, we mention the classification of Hopf hypersurfaces in non-flat complex space forms with constant mean curvature and $\xi$-parallel Ricci tensor provided by Kimura and Maeda in [11]. Next, Maeda in [12], gives the classification of Hopf hypersurfaces in $\mathbb{C} P^{n}, n \geq 3$, with $A \xi=2 \cot (2 r) \xi$ when the focal map $\phi_{r}$ has constant rank on $M$, satisfying $\nabla_{\xi} S=0$, obtaining particular cases of the homogeneous real hypersurfaces in Takagi's list and two kinds of non-homogeneous hypersurfaces. In [13] Suh classified Hopf hypersurfaces in $M^{n}(c), n \geq 2$, whose Ricci tensor is $\eta$-parallel, that is, $g\left(\left(\nabla_{X} S\right) Y, Z\right)=0$, for any $X, Y, Z \in \mathbb{D}$, obtaining real hypersurfaces either of type $(A)$ or of type $(B)$. 
More results on the study of real hypersurfaces in non-flat complex spaces forms in terms of their Ricci tensor are included in Section 6 of [14].

The canonical affine connection defined on a non-degenerate, pseudo-Hermitian CR-manifold is called Tanaka-Webster connection (see $[15,16])$. The generalized Tanaka-Webster connection is a generalization of the previous connection for contact metric manifolds defined by Tanno in [17] and given by

$$
\hat{\nabla}_{X} Y=\nabla_{X} Y+\left(\nabla_{X} \eta\right)(Y) \xi-\eta(Y) \nabla_{X} \xi-\eta(X) \phi Y .
$$

Using the naturally extended affine connection of Tanno's generalized Tanaka-Webster connection, Cho defined the k-th generalized Tanaka-Webster connection $\hat{\nabla}^{(k)}$ for real hypersurfaces $M$ in $M^{n}(c)$ given by

$$
\hat{\nabla}_{X}^{(k)} Y=\nabla_{X} Y+g(\phi A X, Y) \xi-\eta(Y) \phi A X-k \eta(X) \phi Y
$$

for any $X, Y$ tangent to $M$ where $k$ is a nonnull real number (see [18,19]). Then the following relations hold

$$
\hat{\nabla}^{(k)} \eta=0, \quad \hat{\nabla}^{(k)} \xi=0, \quad \hat{\nabla}^{(k)} g=0, \quad \hat{\nabla}^{(k)} \phi=0 .
$$

In particular, if the shape operator of a real hypersurface satisfies $\phi A+A \phi=2 k \phi$, the k-th generalized Tanaka-Webster connection coincides with the Tanaka-Webster connection.

The difference of the Levi-Civita connection and the k-th generalized Tanaka-Webster connection results in a tensor field of type $(1,2)$ given by $F^{(k)}(X, Y)=g(\phi A X, Y) \xi-\eta(Y) \phi A X-k \eta(X) \phi Y$, for any $X, Y$ tangent to $M$ (see [20] Proposition 7.10, pages 234-235). This tensor is called the $k$-th Cho tensor on the real hypersurface $M$. Associated to it, for any $X$ tangent to $M$ and any nonnull real number $k$ the tensor field of type $(1,1) F_{X}^{(k)}$, given by $F_{X}^{(k)} Y=F^{(k)}(X, Y)$ for any $Y \in T M$ can be considered. This operator is named the $k$-th Cho operator corresponding to $X$ and is given by

$$
F_{X}^{(k)} Y=g(\phi A X, Y) \xi-\eta(Y) \phi A X-k \eta(X) \phi Y .
$$

The torsion of the connection $\hat{\nabla}^{(k)}$ is given by $\hat{T}^{(k)}(X, Y)=F_{X}^{(k)} Y-F_{Y}^{(k)} X$ for any $X, Y$ tangent to $M$. Notice that if $X \in \mathbb{D}$, the corresponding k-th Cho operator does not depend on $k$ and is called Cho operator and is simply denoted by $F_{X}$.

Let $T$ be a tensor field of type $(1,1)$ on $M$ and $X$ a vector field tangent to $M$. Then it is easy to see that $\nabla_{X} T=\hat{\nabla}_{X}^{(k)} T$ if and only if $T F_{X}^{(k)}=F_{X}^{(k)} T$. That means that the eigenspaces of $T$ are preserved by $F_{X}^{(k)}$. In [21] we studied the problem of commutativity of Cho operators and shape operator, obtaining that the unique real hypersurfaces in $\mathbb{C} P^{m}, m \geq 3$, such that $F_{X} A=A F_{X}$ for any $X \in \mathbb{D}$ are locally congruent to ruled real hypersurfaces. Similar results were obtained in the case of structure Jacobi operator of real hypersurfaces in $M^{n}(c), n \geq 2$, (see [22,23]).

In this paper we study real hypersurfaces $M$ in $M^{n}(c)$ whose Cho operators commute with the Ricci tensor, i.e.,

$$
F_{X} S=S F_{X}, X \in \mathbb{D} \text {. }
$$

The geometrical meaning is that any eigenspace of the Ricci tensor $S$ is preserved by $F_{X}$. First we prove the following Theorem 1.

Theorem 1. There do not exist Hopf hypersurfaces in $M^{n}(c), n \geq 2$, whose Ricci tensor satisfies relation (2).

Next we study real hypersurfaces in $M^{n}(c), n \geq 2$, which in addition satisfy the relation $h=$ $g(A \xi, \xi)$, where $h=\operatorname{Trace}(A)$ and we obtain the following result (Theorem 2). 
Theorem 2. Let $M$ be a real hypersurface in $M^{n}(c), n \geq 2$, such that $h=g(A \xi, \xi)$. Then $F_{X} S=S F_{X}$ for any $X \in \mathbb{D}$ if and only if $M$ is locally congruent to a ruled real hypersurface.

As a direct consequence of the above Theorem we have Corollary 1.

Corollary 1. There do not exist real hypersurfaces $M$ in $M^{n}(c), n \geq 2$, such that $F_{X}^{(k)} S=S F_{X}^{(k)}$ for any $X$ tangent to $M$ and some nonnull real number $k$, if $h=g(A \xi, \xi)$.

This paper is organized as follows: In Section 2 basic results concerning real hypersurfaces in $M^{n}(c), n \geq 2$, are stated. In Section 3 the proof of Theorem 1 is provided. In Section 4 the proof of Theorem 2 and Corollary are given. At the end of the Section an open problem is stated.

\section{Preliminaries}

In this paper all manifolds, vector fields, etc., will be considered of class $C^{\infty}$ unless otherwise stated. We denote $M$ a connected real hypersurface in a non-flat complex space form, without boundary and $\mathrm{N}$ a locally defined unit normal vector field on it. The Levi-Civita connection of the real hypersurface is denoted by $\nabla$ and $(J, g)$ is the Kählerian structure of ambient space.

For any vector field $X$ tangent to $M$ we write $J X=\phi X+\eta(X) N$ and $-J N=\xi$. Then $(\phi, \xi, \eta, g)$ is an almost contact metric structure on $M$ (see [24]). That is, we have

$$
\phi^{2} X=-X+\eta(X) \xi, \quad \eta(\xi)=1, \quad g(\phi X, \phi Y)=g(X, Y)-\eta(X) \eta(Y)
$$

for any tangent vectors $X, Y$ to $M$. From the above expressions we obtain

$$
\phi \xi=0, \quad \eta(X)=g(X, \xi) .
$$

The complex structure $J$ is parallel and this results in

$$
\left(\nabla_{X} \phi\right) Y=\eta(Y) A X-g(A X, Y) \xi \quad \text { and } \quad \nabla_{X} \xi=\phi A X
$$

for any $X, Y$ tangent to $M$ and $A$ being the shape operator of the immersion. The ambient space has holomorphic sectional curvature $c$. Thus, the equations of Gauss and Codazzi are respectively given by

$$
\begin{array}{r}
R(X, Y) Z=\frac{c}{4}[g(Y, Z) X-g(X, Z) Y+g(\phi Y, Z) \phi X-g(\phi X, Z) \phi Y \\
-2 g(\phi X, Y) \phi Z]+g(A Y, Z) A X-g(A X, Z) A Y
\end{array}
$$

and

$$
\left(\nabla_{X} A\right) Y-\left(\nabla_{Y} A\right) X=\frac{c}{4}[\eta(X) \phi Y-\eta(Y) \phi X-2 g(\phi X, Y) \xi],
$$

for any $X, Y, Z$ tangent to $M$, with $R$ being the curvature tensor of $M$. The (maximal) holomorphic distribution $\mathbb{D}$ on $M$ (if $n \geq 2$ ) is defined at any $P \in M$ by $\mathbb{D}(P)=\left\{X \in T_{P} M \mid g(X, \xi)=0\right\}$.

The above formulas imply that the Ricci tensor on the real hypersurface $M$ is given by

$$
S X=\frac{c}{4}[(2 n+1) X-3 \eta(X) \xi]+h A X-A^{2} X
$$

for any $X$ tangent to $M$, where $h=\operatorname{Trace}(A)$.

In the sequel we need the following result which is owed to Maeda [25] in case of $\mathbb{C} P^{n}, n \geq 2$, and is owed to Montiel [5] in case of $\mathbb{C} H^{n}, n \geq 2$ (also Corollary 2.3 in [14]). 
Theorem 3. Let $M$ be a Hopf hypersurface in $M^{n}(c), n \geq 2$. Then

(i) $\alpha$, which is the principal curvature of the Hopf hypersurface in the direction of $\xi$, is constant.

(ii) If $W$ is a vector field which belongs to $\mathbb{D}$ such that $A W=\lambda W$, then

$$
\left(\lambda-\frac{\alpha}{2}\right) A \phi W=\left(\frac{\lambda \alpha}{2}+\frac{c}{4}\right) \phi W
$$

(iii) If the vector field $W$ satisfies $A W=\lambda W$ and $A \phi W=\nu \phi W$ then

$$
\lambda v=\frac{\alpha}{2}(\lambda+v)+\frac{c}{4}
$$

Remark 1. In case of real hypersurfaces of dimension greater than two the third case of Theorem 3 occurs when $\alpha^{2}+c \neq 0$, since in this case relation $\lambda \neq \frac{\alpha}{2}$ holds.

\section{Proof of Theorem 1}

Let $M$ be a Hopf hypersurface in $M^{n}(c), n \geq 2$, with $A \xi=\alpha \xi$ and whose Ricci tensor satisfies relation (2). Relation (2) taking into account relation (1) is written as

$$
g(\phi A X, S Y) \xi-\eta(S Y) \phi A X=g(\phi A X, Y) S \xi-\eta(Y) S \phi A X
$$

We consider the following two cases:

Case I: $\alpha^{2}+c \neq 0$.

In this case relations of Theorem 3 and Remark 1 hold. Taking $W \in \mathbb{D}$ such that $A W=\lambda W$ then $A \phi W=v \phi W$. Relation (4) due to the previous relations implies

$$
\begin{aligned}
& S \xi=\left[\frac{c}{2}(n-1)+\alpha(h-\alpha)\right] \xi, \quad S W=\left[\frac{c}{4}(2 n+1)+\lambda(h-\lambda)\right] W \text { and } \\
& S \phi W=\left[\frac{c}{4}(2 n+1)+v(h-v)\right] \phi W .
\end{aligned}
$$

Relation (6) for $Y=\xi$ implies

$$
S \phi A X=\left[\frac{c}{2}(n-1)+h \alpha-\alpha^{2}\right] \phi A X, \text { for any } X \in \mathbb{D} .
$$

The above relation for $X=W$ and $X=\phi W$ taking into account relation (7) yields respectively

$$
\lambda\left[\frac{3 c}{4}+h(v-\alpha)-\left(v^{2}-\alpha^{2}\right)\right]=0 \quad v\left[\frac{3 c}{4}+h(\lambda-\alpha)-\left(\lambda^{2}-\alpha^{2}\right)\right]=0
$$

If $\frac{3 c}{4}+h(v-\alpha)-\left(v^{2}-\alpha^{2}\right) \neq 0$ then the first of (8) implies $\lambda=0$ and relation (5) results in $2 \alpha v+c=0$. So we conclude that $M$ has at most three different constant eigenvalues. So $M$ is locally congruent to a real hypersurface of type $(B)$ (see [14]). Substitution of the eigenvalues of these real hypersurfaces in $\lambda=0$ leads to a contradiction.

Therefore, on $M$ we have $\frac{3 c}{4}+h(v-\alpha)-\left(v^{2}-\alpha^{2}\right)=0$. Following similar steps as in the above case we conclude that the second relation of (8) implies $\frac{3 c}{4}+h(\lambda-\alpha)-\left(\lambda^{2}-\alpha^{2}\right)=0$. Combination of the last two relation yields

$$
(v-\lambda)(h-v-\lambda)=0 .
$$

Suppose that $v \neq \lambda$ then $h=\lambda+v$ and relation $\frac{3 c}{4}=\left(v^{2}-\alpha^{2}\right)-h(v-\alpha)$ because of (5) results in $\lambda v=\frac{5 c}{4}+\alpha^{2}$. Substitution of the latter in (5) implies $\alpha(\lambda+v)=2\left(\alpha^{2}+c\right)$. So $\lambda+v$ and $\lambda v$ are constant. Thus, $\lambda, v$ are constant and the real hypersurface has at most three different eigenvalues. So it is locally congruent to a real hypersurface of type $(B)$. Substitution of the eigenvalues of these real hypersurfaces in $\lambda v=\frac{5 c}{4}+\alpha^{2}$ leads to a contradiction. 
Therefore, on $M$ relation $\lambda=v$ holds and this implies that $M$ is locally congruent to a real hypersurface of type $(A)$. So relation (5) becomes

$$
\lambda^{2}=\alpha \lambda+\frac{c}{4}
$$

Furthermore, we have $h=\alpha+(2 n-2) \lambda$. Relation $\frac{3 c}{4}=\left(\lambda^{2}-\alpha^{2}\right)-h(\lambda-\alpha)$ because of the latter results in $c=0$, which is impossible.

Case II: $\alpha^{2}+c=0$.

This case occurs when the ambient space is the complex hyperbolic space $\mathbb{C} H^{n}, n \geq 2$. So we have that $c=-4$ and $\alpha^{2}=4$. Take a unit vector field $W \in \mathbb{D}$ such that $A W=\lambda W$, then $A \phi W=v \phi W$.

First, we suppose that $\lambda \neq \frac{\alpha}{2}$. Then relation (5) owing to $\alpha^{2}-4=0$ yields $v=\frac{\alpha}{2}$ and the real hypersurface has three distinct eigenvalues $\alpha, \lambda$ and $v=\frac{\alpha}{2}$. If $p$ is the multiplicity of $\lambda$ and $q$ is the multiplicity of $v$ we have that $h=\alpha+p \lambda+q v$.

Relation (7) holds. The inner product of the first of relation (7) with $\xi$ implies $\eta(S \xi)=\frac{c}{2}(n-1)+$ $h \alpha-\alpha^{2}$. Moreover, relation (6) for $X=W$ and $Y=\xi$ due to relation (7), $\eta(S \xi)=\frac{c}{2}(n-1)+h \alpha-\alpha^{2}$, $v=\frac{\alpha}{2}$ and $\alpha^{2}=4$ results in $h \lambda=0$.

Suppose that $\lambda \neq 0$ then $h=0$. Moreover, relation (6) for $X=\phi W$ and $Y=\xi$ because of the relation (7) and all the above relations yields $\lambda=-\frac{\alpha}{2}$. Thus, $M$ has three constant principal curvatures. So $M$ is locally congruent to a real hypersurface of type (B). Substitution of the eigenvalues of such real hypersurface in $\lambda=-\frac{\alpha}{2}$ leads to a contradiction.

So $\lambda=0$. Furthermore, relation (6) for $X=\phi W$ and $Y=\xi$ because of relation (7) and all the above relations yields $h=\frac{\alpha}{4}$. The latter due to $h=\alpha+p \lambda+q v$, leads to a contradiction.

Therefore, we conclude that $\lambda=\frac{\alpha}{2}$ will be the only eigenvalue for all vectors in $\mathbb{D}$. In this case the real hypersurface is a horosphere. In the same way as in the previous case we obtain $\eta(S \xi)=$ $\frac{c}{2}(n-1)+h \alpha-\alpha^{2}$. Moreover, relation (6) for $X=W$ and $Y=\xi$ due to $(7), \eta(S \xi)=\frac{c}{2}(n-1)+h \alpha-\alpha^{2}$, $\lambda=\frac{\alpha}{2}$ and $\alpha^{2}=4$ yields $h=0$. In this case we have $h=(2 n-1) \alpha$, so $\alpha=0$ which is impossible and this completes the proof of the Theorem.

\section{Proof of Theorem 2}

In order to prove Theorem 2 the steps below are followed:

- $\quad$ As a consequence of Theorem 1 we conclude Proposition 1.

Proposition 1. There do not exist Hopf hypersurfaces in $M^{n}(c)$, with $h=g(A \xi, \xi)$ and whose Ricci tensor satisfies relation (2).

- Next we study non-Hopf hypersurfaces satisfying the above conditions and the shape operator on $U$ and $\phi U$ orthogonal to $\xi$ is characterized (see Lemma 1). In case of three dimensional real hypersurfaces Lemma 1 leads to the conclusion that the real hypersurface is a ruled one.

- We go on with the study of real hypersurfaces of dimension greater than three. In this case it is proved that the eigenvalues of the shape operator on $\mathbb{D}_{U}$, which consist of the vector fields orthogonal to $\{\xi, U, \phi U\}$, can be:

either all are equal to zero,

or zero and two non-zero $\lambda_{1}$ and $\lambda_{2}$. It is proved that this case can not occur.

Therefore, the only case that occurs is the first one and this leads to the conclusion that $M$ is a ruled real hypersurface.

We are now focused on the study of non-Hopf real hypersurfaces satisfying relation (2) and $h=g(A \xi, \xi)$. In this case also relation (6) holds. First, the scalar product of relation (6) for $Y \in \mathbb{D}$ with $Y$ yields

$$
\eta(S Y) g(\phi A X, Y)=0 \text {, for any } X, Y \in \mathbb{D} \text {. }
$$


Suppose that $g(\phi A X, Y)=0$ for any $X, Y \in \mathbb{D}$. Then $M$ is a ruled hypersurface.

Next we examine the case of $\eta(S Y)=0$, for any $Y \in \mathbb{D}$. The previous relation implies $S \xi=\mu \xi$, for a certain function $\mu$ on $M$. Since $M$ is a non-Hopf real hypersurface we locally have

$$
A \xi=\alpha \xi+\beta U,
$$

where we denote by $\alpha=g(A \xi, \xi), U$ is a unit vector field in $\mathbb{D}, \alpha$ and $\beta$ are functions on $M$ with $\beta \neq 0$. Furthermore, we denote by $\mathbb{D}_{U}$ the orthogonal complementary distribution in $\mathbb{D}$ to the one spanned by $U$ and $\phi U$ (this holds in case of real hypersurfaces with dimension greater than 3).

Lemma 1. Let $M$ be a real hypersurface in $M^{n}(c), n \geq 2$, whose Ricci tensor satisfies relation (2) and $h=\alpha$. Then the shape operator $A$ of $M$ satisfies the relation

$$
A U=\beta \xi \quad A \phi U=0 .
$$

Proof of Lemma 1. Relation (6) for $Y=\xi$ implies $\eta(S \xi) \phi A X=S \phi A X$, for any $X \in \mathbb{D}$. As $S \xi=\mu \xi=$ $\frac{c}{4}(2 n-2) \xi+\alpha A \xi-A^{2} \xi=\left[\frac{c}{4}(2 n-2)\right] \xi-\beta A U$, its scalar product with a vector field $Z$, orthogonal to $\xi$ and $U$, gives $\beta g(A U, Z)=0$. Moreover, the scalar product with $U$ yields $\beta g(A U, U)=0$ from our hypothesis. This implies

$$
A U=\beta \xi .
$$

The scalar product of (6) with $U$ yields $\eta(S Y) g(A \phi U, X)=\eta(Y) g(A \phi S U, X)$. Taking $Y=\xi$ it becomes

$$
\eta(S \xi) g(A \phi U, X)=g(A \phi S U, X)
$$

for any $X \in \mathbb{D}$. Since $S U=\left(\frac{c}{4}(2 n+1)-\beta^{2}\right) U$ and $\eta(S \xi)=\frac{c}{4}(2 n-2)-\beta^{2}$, from (11) we have

$$
A \phi U=0 .
$$

From now on we suppose that the dimension of the real hypersurface is greater than 3 . From Lemma 1 we know now that $\mathbb{D}_{U}$ is $A$-invariant. Take now a unit $Y \in \mathbb{D}_{U}$ such that $A Y=\lambda Y$. From (6) we get $\lambda(g(\phi Y, S Z) \xi-\eta(S Z) \phi Y)=\lambda(g(\phi Y, Z) S \xi-\eta(Z) S \phi Y)$, for any Z tangent to $M$. Therefore either $\lambda=0$, or, if $\lambda \neq 0$, taking $Z=\xi$, we have $S \phi Y=\eta(S \xi) \phi Y$.

Now if $A Y=0$ for any $Y \in \mathbb{D}_{U}$ we obtain a ruled real hypersurface.

Let us suppose that $A Y=0$. Then $S Y=\frac{c}{4}(2 n+1) Y$. For any $X \in \mathbb{D}$ it follows $\frac{c}{4}(2 n+1) g(\phi A X, Y) \xi=g(\phi A X, Y) S \xi$. Therefore, for any $X \in \mathbb{D},\left[\frac{c}{4}(2 n+1)-\eta(S \xi)\right] g(\phi A X, Y)=0$. As $\frac{c}{4}(2 n+1)-\eta(S \xi)=\frac{3 c}{4}+\beta^{2} \neq 0$, we obtain $A \phi Y=0$. If we denote by $T_{0}$ the distribution in $\mathbb{D}_{U}$ corresponding to the eigenvalue 0 , we have that $T_{0}$ is $\phi$-invariant. Thus the complementary distribution of $T_{0}$ in $\mathbb{D}_{U}$ is also $\phi$-invariant.

Let $\left\{E_{1}, \ldots E_{2 p}\right\}$ be an orthonormal basis of eigenvectors in the complementary distribution. Then relation $S \phi Y=\eta(S \xi) \phi Y$ implies for any $i=1, \ldots, 2 p S \phi E_{i}=\eta(S \xi) \phi E_{i}$. As $\left\{\phi E_{1}, \ldots, \phi E_{2 p}\right\}$ is also an orthonormal basis of the distribution, we obtain that for any $X \in \mathbb{D}_{U}$ such that $A X \neq 0$, $S X=\eta(S \xi) X$. If $X$ is an eigenvector with eigenvalue $\lambda \neq 0$, it follows $S X=\left[\frac{c}{4}(2 n+1)+\alpha \lambda-\lambda^{2}\right] X=$ $\left[\frac{c}{4}(2 n-2)-\beta^{2}\right] X$. This yields

$$
\frac{3 c}{4}+\lambda(\alpha-\lambda)+\beta^{2}=0 .
$$


Relation (12) implies that the unique possible nonnull eigenvalues in $\mathbb{D}_{U}$ are $\lambda_{1}=\frac{\alpha}{2}+$ $\sqrt{\left(\frac{\alpha}{2}\right)^{2}+\frac{3 c}{4}+\beta^{2}}$ and $\lambda_{2}=\frac{\alpha}{2}-\sqrt{\left(\frac{\alpha}{2}\right)^{2}+\frac{3 c}{4}+\beta^{2}}$. If $\lambda_{2}$ does not appear, as $h=\alpha$ and if $p$ is the multiplicity of $\lambda_{1}$ relation $h=\alpha$ results in

$$
\alpha=\alpha+p\left(\frac{\alpha}{2}+\sqrt{\left(\frac{\alpha}{2}\right)^{2}+\frac{3 c}{4}+\beta^{2}}\right) .
$$

Similarly, if $\lambda_{1}$ does not appear and $q$ is the multiplicity of $\lambda_{2}$

$$
\alpha=\alpha+q\left(\frac{\alpha}{2}-\sqrt{\left(\frac{\alpha}{2}\right)^{2}+\frac{3 c}{4}+\beta^{2}}\right) .
$$

Combining relations (13) and (14) yields $\frac{\alpha}{2}= \pm \sqrt{\left(\frac{\alpha}{2}\right)^{2}+\frac{3 c}{4}+\beta^{2}}$, and this results in $\left(\frac{\alpha}{2}\right)^{2}=\left(\frac{\alpha}{2}\right)^{2}+\frac{3 c}{4}+\beta^{2}$. In case the ambient space is $\mathbb{C} P^{n}$ the previous relation is impossible. In case the ambient space is $\mathbb{C} H^{n}$ the previous relation implies $\beta^{2}=-\frac{3 c}{4}$ and since $c=-4$ we obtain $\lambda_{1}=\frac{\alpha}{2}+\sqrt{\left(\frac{\alpha}{2}\right)^{2}}=\alpha$ and $\lambda_{2}=\frac{\alpha}{2}-\sqrt{\left(\frac{\alpha}{2}\right)^{2}}=0$. Thus $p \alpha=0$. Therefore, either $p=0$ and $M$ is ruled or $\alpha=0$, which implies $h=0$. So $M$ is ruled and minimal.

From now on we suppose that both of the eigenvalues $\lambda_{1}$ and $\lambda_{2}$ do appear as eigenvalues in $\mathbb{D}_{U}$. Furthermore, suppose that there exists $Y \in \mathbb{D}_{U}$ such that $A Y=A \phi Y=0$. From the Codazzi equation $\left(\nabla_{Y} A\right) \xi-\left(\nabla_{\xi} A\right) Y=-\frac{c}{4} \phi Y$. Developing it we get $Y(\alpha) \xi+Y(\beta) U+\beta \nabla_{Y} U+A \nabla_{\xi} Y=-\frac{c}{4} \phi Y$. Its scalar product with $\xi$ yields

$$
\Upsilon(\alpha)+\beta g\left(\nabla_{\xi} Y, U\right)=0
$$

and its scalar product with $U$ gives

$$
Y(\beta)=0 .
$$

Let $Z \in \mathbb{D}_{U}$ such that $A Z=\lambda Z$ (where either $\lambda=\lambda_{1}$ or $\lambda=\lambda_{2}$ ). As above, $\left(\nabla_{Z} A\right) \xi-$ $\left(\nabla_{\xi} A\right) Z=-\frac{c}{4} \phi Z$ implies $Z(\alpha) \xi+\alpha \phi A Z+Z(\beta) U+\beta \nabla_{Z} U-A \phi A Z-(\xi)(\lambda) Z-\lambda \nabla_{\xi} Z+A \nabla_{\xi} Z=$ $-\frac{c}{4} \phi Z$. Its scalar product with $\xi$ implies

$$
Z(\alpha)+\beta g\left(\nabla_{\xi} Z, U\right)=0
$$

and its scalar product with $U$ yields

$$
Z(\beta)-\lambda g\left(\nabla_{\xi} Z, U\right)=0
$$

From (17) and (18) we have

$$
\lambda Z(\alpha)+\beta Z(\beta)=0 .
$$

Moreover, $\left(\nabla_{Z} A\right) U-\left(\nabla_{U} A\right) Z=0$ yields $Z(\beta) \xi+\beta \phi A Z-A \nabla_{Z} U-U(\lambda) Z-\lambda \nabla_{U} Z+$ $A \nabla_{U} Z=0$. Taking its scalar product with $\xi$ we obtain

$$
Z(\beta)+\beta g\left(\nabla_{U} Z, U\right)=0
$$

and its scalar product with $U$ gives

$$
\lambda g\left(\nabla_{U} Z, U\right)=0 .
$$

As $\lambda \neq 0$, from (20) and (21) we have $Z(\beta)=0$ and from (19)

$$
Z(\alpha)=Z(\beta)=0 .
$$


On the other hand, $\left(\nabla_{\xi} A\right) U-\left(\nabla_{U} A\right) \xi=\frac{c}{4} \phi U$ implies $\xi(\beta) \xi+\beta \phi A \xi-A \nabla_{\xi} U-U(\alpha) \xi-$ $U(\beta) U-\beta \nabla_{U} U=\frac{c}{4} \phi U$. Its scalar product with $\xi$ yields $\xi(\beta)-U(\alpha)=0$ and the scalar product with $U$ implies $U(\beta)=0$. Therefore

$$
\xi(\beta)=U(\alpha) \quad U(\beta)=0 .
$$

Analogously, developing $\left(\nabla_{\xi} A\right) \phi U-\left(\nabla_{\phi U} A\right) \xi=-\frac{c}{4} U$ and taking its scalar product with $\xi$, respectively with $U$, we obtain

$$
(\phi U)(\alpha)=\alpha \beta-\beta g\left(\nabla_{\xi} \phi U, U\right)
$$

and

$$
(\phi U)(\beta)=\beta^{2}+\frac{c}{4} .
$$

Let $p$ be the multiplicity of $\lambda_{1}$ and $q$ the multiplicity of $\lambda_{2}$. As $h=\alpha$ we have $(p+q) \frac{\alpha}{2}+(p-$ q) $\sqrt{\left(\frac{\alpha}{2}\right)^{2}+\beta^{2}+\frac{3 c}{4}}=0$. As $U(\beta)=0$, differentiating the latter with respect to $U$ we get $\left(\frac{p+q}{2}+\right.$ $\left.\frac{p-q}{4 \sqrt{\left(\frac{\alpha}{2}\right)^{2}+\beta^{2}+\frac{3 c}{4}}} \alpha\right) U(\alpha)=0$. If we suppose $U(\alpha) \neq 0$, then we have $2(p+q) \sqrt{\left(\frac{\alpha}{2}\right)^{2}+\beta^{2}+\frac{3 c}{4}}=(q-p) \alpha$. This yields $\left((p+q)^{2}-(q-p)^{2}\right) \alpha^{2}+(p+q)^{2}\left(4 \beta^{2}+3 c\right)=0$. Taking the derivative of this expression in the direction of $U$ we get $2 \alpha\left((p+q)^{2}-(q-p)^{2}\right) U(\alpha)=0$, and as we are supposing $U(\alpha) \neq 0$ the fact that $(p+q)^{2}-(q-p)^{2}=4 p q \neq 0$ yields $\alpha=0$. This contradicts $U(\alpha) \neq 0$, and we have proved that $U(\alpha)=0$. So the first of (23) yields

$$
U(\alpha)=\xi(\beta)=0 .
$$

Following similar steps it is proved that $\xi(\alpha)=0$.

Relations (16), (22), (23) and (26) result in

$$
\operatorname{grad}(\beta)=\left(\beta^{2}+\frac{c}{4}\right) \phi U
$$

As $g\left(\nabla_{X} \operatorname{grad}(\beta), Y\right)=g\left(\nabla_{Y} \operatorname{grad}(\beta), X\right)$ for any $X, Y$ tangent to $M$, we have $X\left(\beta^{2}+\frac{c}{4}\right) g(\phi U, Y)+$ $\left(\beta^{2}+\frac{c}{4}\right) g\left(\nabla_{X} \phi U, Y\right)=Y\left(\beta^{2}+\frac{c}{4}\right) g(\phi U, X)+\left(\beta^{2}+\frac{c}{4}\right) g\left(\nabla_{Y} \phi U, X\right)$, for any $X, Y$ tangent to $M$. Taking $X=\xi$ we obtain $\left(\beta^{2}+\frac{c}{4}\right)\left[g\left(\nabla_{\xi} \phi U, Y\right)+g(U, A Y)\right]=0$ for any $Y$ tangent to $M$.

Suppose that $g\left(\nabla_{\xi} \phi U, Y\right)+g(U, A Y) \neq 0$ then the above relation implies $\beta^{2}+\frac{c}{4}=0$, This case occurs when the ambient space is the complex hyperbolic space. So we have that the nonnull eigenvalues in $\mathbb{D}_{U}$ are $\lambda_{1}=\frac{\alpha}{2}+\sqrt{\left(\frac{\alpha}{2}\right)^{2}+\frac{c}{2}}$ and $\lambda_{2}=\frac{\alpha}{2}-\sqrt{\left(\frac{\alpha}{2}\right)^{2}+\frac{c}{2}}$ with multiplicity $p$ and $q$ respectively. Since, $h=\alpha$ we obtain $4 p q\left(\frac{\alpha}{2}\right)^{2}=\frac{c}{2}(q-p)^{2}$, which is a contradiction, since $c<0$.

So on $M$, we have $g\left(\nabla_{\xi} \phi U, Y\right)=-g(U, A Y)$ for any $Y$ tangent to $M$. If $Y=U$ it follows $g\left(\nabla_{\xi} \phi U, U\right)=0$ and from $(24)$

$$
(\phi U)(\alpha)=\alpha \beta .
$$

Moreover, from the above relation we also know that $g\left(\nabla_{\xi} \phi U, \phi Y\right)=-g(U, A \phi Y)$ for any $Y$ tangent to $M$. If $Y \in \mathbb{D}_{U}$ satisfies $A Y=A \phi Y=0$, this and (3) yield $g\left(\nabla_{\xi} U, Y\right)=0$ and from (15) we get

$$
Y(\alpha)=0 .
$$

From Equations (22), (25), (26), (28) and (29) we assure

$$
\operatorname{grad}(\alpha)=\alpha \beta \phi U .
$$


Recall that $(p+q) \frac{\alpha}{2}+(p-q) \sqrt{\left(\frac{\alpha}{2}\right)^{2}+\beta^{2}+\frac{3 c}{4}}=0$. Taking its derivative in the direction of $\phi U$ and bearing in mind (27) and (28) we obtain $\frac{p+q}{2} \alpha \beta+\frac{p-q}{2 \sqrt{\left(\frac{\alpha}{2}\right)^{2}+\beta^{2}+\frac{3 c}{4}}}\left(\frac{1}{2} \alpha^{2} \beta+2 \beta\left(\beta^{2}+\frac{c}{4}\right)\right)=0$. From this we arrive to

$$
\left((p+q)^{2}-(q-p)^{2}\right) \frac{\alpha^{4}}{4}+(p+q)^{2} \alpha^{2}\left(\frac{3 c}{4}+\beta^{2}\right)=(q-p)^{2}\left(\beta^{2}+\frac{c}{4}\right)\left(2 \alpha^{2}+4 \beta^{2}+c\right) .
$$

Derivating (31) in the direction of $\phi$ Uand bearing in mind (27) and (28) we obtain

$$
\left((p+q)^{2}-(q-p)^{2}\right) \alpha^{4}+2(p+q)^{2} \alpha^{2}\left(2 \beta^{2}+c\right)=4(q-p)^{2}\left(\beta^{2}+\frac{c}{4}\right)\left(2 \alpha^{2}+4 \beta^{2}+c\right) .
$$

From (31) and (32) it follows $c(p+q)^{2} \alpha^{2}=0$. This yields $\alpha=0$.

Relation (31) gives

$$
4(q-p)^{2}\left(\beta^{2}+\frac{c}{4}\right)^{2}=0 .
$$

Suppose that $p \neq q$ then the above relation implies $\beta^{2}+\frac{c}{4}=0$. This case occurs when the ambient space is complex hyperbolic space and the nonnull eigenvalues in $\mathbb{D}_{U}$ are $\lambda_{1}=\sqrt{\frac{c}{2}}$ and $\lambda_{2}=-\sqrt{\frac{c}{2}}$, which is a contradiction, since $c<0$.

Therefore, on $M$ we have $p=q$ and $\mathbb{D}_{U}$ can be written as follows

$$
\mathbb{D}_{U}=T_{0} \bigoplus T_{\sqrt{\beta^{2}+\frac{3 c}{4}}} \bigoplus_{-\sqrt{\beta^{2}+\frac{3 c}{4}}}
$$

and the last two eigenspaces have the same dimension.

Let $\left\{Z_{1}, \ldots, Z_{p}\right\}$ an orthonormal basis of $T \sqrt{\beta^{2}+\frac{3 c}{4}}$. Take $i, j \in\{1, \ldots, p\}, i \neq j$ (we suppose that $p \geq 2)$. The Codazzi equation yields $\left(\nabla_{Z_{i}} A\right) Z_{j}-\left(\nabla_{Z_{j}} A\right) Z_{i}=-\frac{c}{2} g\left(\phi Z_{i}, Z_{j}\right) \xi$. As $\beta$ is constant along the directions in $T \sqrt{\beta^{2}+\frac{3 c}{4}}$ we obtain

$$
\sqrt{\beta^{2}+\frac{3 c}{4}} \nabla_{Z_{i}} Z_{j}-A \nabla_{Z_{i}} Z_{j}-\sqrt{\beta^{2}+\frac{3 c}{4}} \nabla_{Z_{j}} Z_{i}+A \nabla_{Z_{j}} Z_{i}=-\frac{c}{2} g\left(\phi Z_{i}, Z_{j}\right) \xi
$$

Its scalar product with $\xi$ yields

$$
\beta g\left(\left[Z_{j}, Z_{i}\right], U\right)=\frac{c}{2}\left(\beta^{2}+\frac{c}{2}\right) g\left(\phi Z_{i}, Z_{j}\right)
$$

and its scalar product with $U$ implies

$$
g\left(\left[Z_{j}, Z_{i}\right], U\right)=\frac{c}{2} \beta g\left(\phi Z_{i}, Z_{j}\right) .
$$

From (33) and (34) we obtain $g\left(\phi Z_{i}, Z_{j}\right)=0$. This means that for any $Z \in T_{\sqrt{\beta^{2}+\frac{3 c}{4}}}, \phi Z \in$ $T_{-\sqrt{\beta^{2}+\frac{3 c}{4}}}$. Call $\lambda=\sqrt{\beta^{2}+\frac{3 c}{4}}$. Take $Z \in T_{\lambda}$. The Codazzi equation yields $-\lambda \nabla_{Z} \phi Z-A \nabla_{Z} \phi Z-$ $\lambda \nabla_{\phi Z} Z+A \nabla_{\phi Z} Z=-\frac{c}{2} \xi$. Its scalar product with $\xi$ yields

$$
\beta g\left(\nabla_{\phi Z} Z, U\right)-\beta g\left(\nabla_{Z} \phi Z, U\right)=-\frac{c}{2}\left(\beta^{2}+c\right)
$$

and its scalar product with $U$, bearing in mind that $\lambda \neq 0$, gives

$$
g\left(\nabla_{\phi Z} Z, U\right)+g\left(\nabla_{Z} \phi Z, U\right)=0 .
$$


From (35) and (36) we obtain

$$
g\left(\nabla_{Z} \phi Z, U\right)=-g\left(\nabla_{\phi Z} Z, U\right)=\frac{\beta^{2}+c}{\beta} .
$$

On the other hand $\left(\nabla_{\phi U} A\right) \phi Z-\left(\nabla_{\phi Z} A\right) \phi U=0$. This yields $-(\phi U)(\lambda) \phi Z-\lambda \nabla_{\phi U} \phi Z-$ $A \nabla_{\phi U} \phi Z+A \nabla_{\phi Z} \phi U=0$. Its scalar product with $\phi Z$ yields $-(\phi U)(\lambda)-\lambda g\left(\nabla_{\phi Z} \phi U, \phi Z\right)=0$. From (3) $g\left(\nabla_{\phi Z} \phi U, \phi Z\right)=g\left(\nabla_{\phi Z} U, Z\right)$. Bearing in mind the value of $\lambda$, from (37) it follows $\beta^{2}\left(\beta^{2}+c\right)+\left(\beta^{2}+\frac{3 c}{4}\right)\left(\beta^{2}+c\right)=0$. That is, $\beta^{4}+\frac{5 c}{2} \beta^{2}+\frac{3 c^{2}}{4}=0$. Thus $\beta$ is constant and this results in $\operatorname{grad}(\beta)=0$. So relation (27) implies $\beta^{2}+\frac{c}{4}=0$, which occurs in case the ambient space is $\mathbb{C} H^{n}$. In this case, substitution of the last one in $\beta^{4}+\frac{5 c}{2} \beta^{2}+\frac{3 c^{2}}{4}=0$ implies $c=0$, which is impossible. This means that our non Hopf real hypersurfaces must be ruled and this completes the proof of Theorem 2.

In order to prove the Corollary, suppose that $M$ is a ruled real hypersurface such that for some nonnull $k, F_{\xi}^{(k)} S Y=S F_{\xi}^{(k)} Y$ for any $Y$ tangent to $M$. The previous relation because of $F_{X}^{(k)} Y=$ $g(\phi A X, Y) \xi-\eta(Y) \phi A X-k \eta(X) \phi Y$ becomes

$$
g(\phi A \xi, S Y) \xi-\eta(S Y) \phi A \xi-k \phi S Y=g(\phi A \xi, Y) S \xi-\eta(Y) S \phi A \xi-k S \phi Y
$$

for any $Y$ tangent to $M$.

The shape operator of a ruled real hypersurface $M$ is given by

$$
A \xi=\alpha \xi+\beta U, \quad A U=\beta \xi \text { and } A Y=0, \quad \text { for any } Y \text { orthogonal to }\{\xi, U\} .
$$

The Ricci tensor (4) for $X=\xi, X=U$ and $X=Y$, where $Y$ is any orthogonal vector to $\{\xi, U\}$, because of $h=g(A \xi, \xi)=\alpha$ and relation (39) becomes respectively

$$
S \xi=\left(\frac{c}{2}(n-1)-\beta^{2}\right) \xi, \quad S U=\frac{c}{4}(2 n+1) U-\beta^{2} \xi \quad \text { and } \quad S Y=\frac{c}{4}(2 n+1) Y .
$$

Relation (38) for $Y=U$ bearing in mind the first of relation (39) and the second of relation (40) leads to $\beta=0$, which is a contradiction since $M$ is ruled and this completes the proof of the Corollary.

\section{Discussion}

If in our Theorem we suppose $h \neq g(A \xi, \xi)$, it is easy to see that $\beta^{2}=g(A \xi, \xi)(h-g(A \xi, \xi))-3$ for a non Hopf real hypersurface. This might produce a new kind of real hypersurfaces.

Conjecture (open problem): Such real hypersurfaces in complex space forms do not exist.

Author Contributions: Writing—original draft, G.K., K.P. and J.D.P.; writing—review \& editing, G.K., K.P. and J.D.P. All authors contributed equally to this research and in writing the paper. All authors have read and agreed to the published version of the manuscript.

Funding: This research received no external funding.

Acknowledgments: The authors would like to thank the reviewers for their valuable comments in order to improve the paper.

Conflicts of Interest: The authors declare no conflict of interest.

\section{References}

1. Kimura, M. Real hypersurfaces and complex submanifolds in complex projective space. Trans. Am. Math. Soc. 1986, 296, 137-149. [CrossRef]

2. Takagi, R. On homogeneous real hypersurfaces in a complex projective space. Osaka J. Math. 1973, 10, 495-506.

3. Takagi, R. Real hypersurfaces in complex projective space with constant principal curvatures. J. Math. Soc. Jpn. 1975, 27, 43-53. [CrossRef] 
4. Takagi, R. Real hypersurfaces in complex projective space with constant principal curvatures II. J. Math. Soc. Jpn. 1975, 27, 507-516. [CrossRef]

5. Montiel, S. Real hypersurfaces of a complex hyperbolic space. J. Math. Soc. Jpn. 1985, 35, 515-535. [CrossRef]

6. Berndt, J. Real hypersurfaces with constant principal curvatures in complex hyperbolic space. J. Reine Angew. Math. 1989, 395, 132-141. [CrossRef]

7. Kimura, M. Sectional curvatures of holomorphic planes of a real hypersurface in $P^{n}(\mathbb{C})$. Math. Ann. 1987, 276, 487-497. [CrossRef]

8. Lohnherr, M.; Reckziegel, H. On ruled real hypersurfaces in complex space forms. Geom. Dedicata 1999, 74, 267-286. [CrossRef]

9. Ki, U.H. Real hypersurfaces with parallel Ricci tensor of a complex space form. Tsukuba J. Math. 1989, 13, 73-81. [CrossRef]

10. Kim, U.K. Nonexistence of Ricci-parallel real hypersurfaces in $\mathbb{C} P^{2}$ and $\mathbb{C} H^{2}$. Bull. Korean Math. Soc. 2004, 41, 699-708. [CrossRef]

11. Kimura, M.; Maeda, S. On real hypersurfaces of a complex projective space II. Tsukuba J. Math 1991, 15, 547-561. [CrossRef]

12. Maeda, S. Ricci tensors of real hypersurfaces in a complex projective space. Proc. Am. Math. Soc. 1994, 122, 1229-1235. [CrossRef]

13. Suh, Y.J. On real hypersurfaces of a complex space form with $\eta$-parallel Ricci tensor. Tsukuba J. Math. 1990, 14, 27-37. [CrossRef]

14. Niebergall, R.; Ryan, P.J. Real hypersurfaces in complex space forms. Tight Taut Submanifolds Msri Publ. 1997, 32, 233-305.

15. Tanaka, N. On non-degenerate real hypersurfaces, graded Lie algebras and Cartan connections. Jpn. J. Math. 1976, 2, 131-190. [CrossRef]

16. Webster, S.M. Pseudohermitian structures on a real hypersurface. J. Diff. Geom. 1978, 13, 25-41. [CrossRef]

17. Tanno, S. Variational problems on contact Riemennian manifolds. Trans. Am. Math. Soc. 1989, 314, 349-379. [CrossRef]

18. Cho, J.T. CR-structures on real hypersurfaces of a complex space form. Publ. Math. Debr. 1999, 54, 473-487.

19. Cho, J.T. Pseudo-Einstein CR-structures on real hypersurfaces in a complex space form. Hokkaido Math. J. 2008, 37, 1-17. [CrossRef]

20. Kobayashi, S.; Nomizu, K. Foundations on Differential Geometry; Interscience: New York, NY, USA, 1963; Volume 1.

21. Pérez, J.D.; Suh, Y.J. Generalized Tanaka-Webster and covariant derivatives on a real hypersurface in a complex projective space. Monatsh. Math. 2015, 177, 637-647. [CrossRef]

22. Panagiotidou, K.; Pérez, J.D. Commuting conditions of the $k$-th Cho operator with the structure Jacobi operator of real hypersurfaces in complex space forms. Open Math. 2015, 13, 321-332. [CrossRef]

23. Pérez, J.D. Commutativity of Cho and structure Jacobi operators of a real hypersurface in a complex projective space. Ann. Mat. Pura Appl. 2015, 194, 1781-1794. [CrossRef]

24. Blair, D.E. Riemannian Geometry of Contact and Symplectic Manifolds; Progress in Mathematics 203; Birkhauser Boston Inc.: Boston, MA, USA, 2002.

25. Maeda, Y. On real hypersurfaces of a complex projective space. J. Math. Soc. Jpn. 1976, 28, 529-540. [CrossRef]

(C) 2020 by the authors. Licensee MDPI, Basel, Switzerland. This article is an open access article distributed under the terms and conditions of the Creative Commons Attribution (CC BY) license (http:/ / creativecommons.org/licenses/by/4.0/). 